\title{
Orientierungshilfe für Konsum
}

\author{
Im Auftrag der IKEA-Stiftung, Hofheim-Wallau, hat das IÖW eine vergleichen- \\ de Übersicht über produktbezogene Kennzeichen erstellt. Ziel der Untersuchung \\ war es, eine ausgewählte Anzahl von Labels zu beschreiben und zu bewerten.
}

Von Gerd Scholl

$\mathrm{D}$ ie Zahl der Verbraucherinnen und Verbraucher, die bei ihren Kaufentscheidungen zusehends ökologische, soziale oder andere Aspekte berücksichtigen, wächst von Tag zu Tag. Als Reaktion dieser veränderten Präferenzen auf der Nachfrageseite sind mittlerweile viele Hersteller dazu übergegangen ihre Produkte unter ökologischen oder sozialen Aspekten zu optimieren. Die anschauliche Informationsaufbereitung mittels Kennzeichen ist ein möglicher Weg den Zusatznutzen „Umweltfreundlichkeit“ oder „Sozialverträglichkeit“, den die Käufer neben der Gebrauchseigenschaft des Produktes erwerben, am Markt transparent zu machen.

Doch gerade dieser Wunsch nach Markttransparenz hat sich mittlerweile in sein Gegenteil verkehrt. Fast jedes Produkt ziert mittlerweile ein Label, welches seine besonderen technischen, gesundheitlichen, sozialen Eigenschaften oder seine Umweltqualitäten hervorhebt. Die ursprüngliche Informationshilfe droht also allmählich in einer nicht mehr überschaubaren Flut unterschiedlicher Labels - Experten gehen von über 1000 Kennzeichen aus - unterzugehen.

\section{Systematische Einordnung}

Bei der Darstellung und Bewertung der Produktkennzeichen stehen drei Fragen im Vordergrund: Welche Institutionen bzw. Unternehmen vergaben die Produktlabels? Nach welchen Kriterien und Richtlinien werden die Label vergeben? Wie verläuft der Prozeß der Erarbeitung von Kriterien und die Ausgestaltung und Dokumentation des Vergabeprozesses? Diese drei Dimensionen dienten als Grundlage zur Strukturierung von zweiseitigen, standardisierten Datenblättern, die für 32 Kennzeichen erstellt wurden. Sie enthalten komprimierte Informationen und einen kurzen Kommentar. Neben Universalkennzeichen wie dem „Blauen Engel“ oder der Kennzeichnung der Stiftung Warentest, die für mehrere Produkte vergeben werden, waren insbesondere Textil- und Teppich- sowie
Lebensmitelkennzeichen stark vertreten. Die Spannbreite reichte von firmenindividuellen Logos (z.B. Steilmann „It's one world“) bis hin $\mathrm{zu}$ Siegeln, die unter staatlicher Beteiligung verliehen werden (z.B. „Öko-Punkt Sachsen“).

\section{Bewertung von Bewertungen}

Bei der Beurteilung der einzelnen Labels handelt es sich um eine „Bewertung von Bewertungen“ die nicht immer eindeutig zu treffen war. Labels zeichnen nämlich zum einen sehr unterschiedliche Produkte aus - einerseits Kosmeti$\mathrm{ka}$, andererseits Urlaubsstrände - und zum anderen sehr unterschiedliche Produktqualitäten. So zertifiziert das „Rugmark“-Label beispielsweise soziale Aspekte der Teppichherstellung (,ohne Kinderarbeit"), wohingegen das „Teppich Siegel“ für schadstoffarme und besonders strapazierfähige Teppichböden steht.

Die Beurteilung wurde deshalb auf einzelne Produktgruppen eingeschränkt. Sie ist differenziert nach den beiden Rubriken „Inhaltlicher Anspruch" - in ökologischer, sozialer, technischer oder anderer Art - und „Glaubwürdigkeit“ - Status des Zeichengebers und Vergabeprozeß.; „Pluralität“, „Unabhängigkeit“, „Kontrolle“ oder „Transparenz“. Neben den zahlreichen umweltorientierten Labels läßt sich ein Trend in Richtung sozialbezogene Kennzeichnung ausmachen. Was mit „traditionellen“ Kennzeichen, die teilweise aus der entwicklungspolitischen Diskussion hervorgegangen sind, wie dem „TransFair"-Siegel für Kaffee (Unterstützung kleinbäuerlicher Strukturen in den Anbauländern) oder dem „Rugmark"-Label begonnen hat, scheint sich fortzusetzen. Beispiele dafür sind das Tourismus-Label „Blaue Schwalbe““، das die Einhaltung tariflicher Mindeststandards und den Verzicht auf Schwarzarbeit fordert, oder das Textillabel „Ecoproof“, das vom TÜV Rheinland vergeben wird und Kinderarbeit untersagt. Dieser Trend zeigt die zunehmende Bedeutung sozialer Aspekte eines nachhaltigen Konsums an. Produktlabels können hierzu einen Beitrag leisten, indem sie Verbraucher für die ökologischen und sozialen Eigenschaften von Produkten sensibilisieren.

\section{Anmerkung}

Die Studie "Darstellung und Bewertung umwelt- und sozialbezogener Kennzeichen" ist beim Bundesverband für Umweltberatung, Bremen, zum Preis von 30 Mark erhältlich. Tel. (0421) 343400, Fax 3499267.

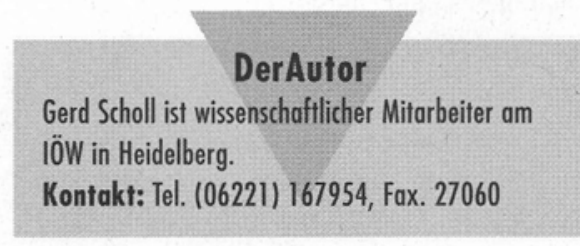

\section{Buchtip}

Cornelia Weskamp (Hg): Ökologischer Konsum. Ansätze und Leitbilder für eine ökologisch und sozialverträgliche Lebensweise. IÖW 1996, 136 Seiten, 26 Mark

Vom 7. bis 9. Dezember 1994 veranstaltete das Ökoforums eine Tagung mit dem Titel: „Geld oder leben !? Ökologischer Konsum: Anspruchshaltung oder Genügsamkeit?". Auf der Basis der auf der Tagung gehaltenen Referate und ergänzt durch weitere Beiträge ist ein Buch erschienen, das versucht, die Entwicklung des neuen Leitbildes "Nachaltiger Konsum" nachzuzeichnen. Der thematische Teil des Buches beginnt mit einem Beitrag von Cornelia Weskamp zu den Determinanten ökologischen Konsums. Sie gibt hierin einen Überblick zu derzeitigen Forschungsansätzen und weist ouf mögliche Handlungsfelder hin. Irmgard Schultz zeigt Leitbilder der Konsumpolitik ouf und von Christoph Ewen erörtert mit Hilfe Ehrlich-Formel den Zusammenhang von Konsum und Umweltzerstörung.
Birgit Soete und Jürgen Meyerhoff behandeln Internationalen Handel und ökologischen Konsum. Weitere Inhalte sind: Gerd Scholl: Die Dematerialisierung des Konsums - ein schöner Troum; Ines Weller: Zum wechselseitigen Zusammenhang zwischen Ökologisierung des Konsums und der Produktion von Bekleidung; Willi Sieber: Erst geht die Kuh und dann der Gast? Sanfter Tourismus oder Urlaub für alle?, Willi Loose: Wohnen ohne (eigenes) Auto; Helmut Pörnbacher: Nachhaltigkeit im Supermarkt; Volrad Wollny: Effizienz, Suffizienz und regionaler Konsum am Beispiel der Verpackungen; Frank Waskow: Nachwachsende Rohstoffe am Beispiel der Nutzplanze Hanf. Im zweiten Teil über das Ökoforum folgt eine retrospektive Betrachtung der Gründung von Ökoforum und ein Beitrag von Irene Reifenhöuser und Martino Schott zur Bedeutung von Ökoforum-Projekten. Schließlich enthält der Band noch die Selbstdarstellungen des Ökoforum und der angeschlossenen Institute. 
(c) 20I0 Authors; licensee IÖW and oekom verlag. This is an article distributed under the terms of the Creative Commons Attribution Non-Commercial No Derivates License (http://creativecommons.org/licenses/by-nc-nd/3.o/), which permits unrestricted use, distribution, and reproduction in any medium, provided the original work is properly cited. 